\title{
DRN: A CORBA-Based Distributed Resource Navigation System for Data Grid
}

\author{
Jia-Jia Miao, Ai-Ping Li, Jia Yan, and Quan-Yuan Wu \\ School of Computer Science, National University of Defense Technology \\ Changsha 4100073, P.R. China \\ elanmiao@yahoo.com.cn, apli@x263.net, elanmiao@gmail.com
}

\begin{abstract}
Data grids are becoming increasingly important for sharing distributed data and information in collaborative environments. This paper presents a CORBA-based Distributed Resource Navigation system consisting of Resource Agents and Navigation Service. The former one is a middleware that provides applications with a uniform set of operations to access heterogeneous distributed storage resources including file systems and database systems, and archival storage systems. The latter one is a metadata navigation service which is utilized by DRN to improve data publishing and lookup efficiency. Experiments show that CORBA-based DRN can provide the essential data virtualization services, and that Navigation service and Resource agent have a good performance with concurrent accesses.
\end{abstract}

Keywords: CORBA, Data intensive computing, Data virtualization.

\section{Introduction}

Grids [1] are environments that enable software applications to integrate computational and data resources that are managed by diverse organizations in widespread locations. Examples of data-intensive applications include experimental analyses and simulations in several scientific disciplines [2,5], such as high-energy physics, climate modeling, biomedical applications, earthquake engineering, oceanographic systems, and astronomy and so on.

CORBA has emerged as a popular distributed computing standard and meets the necessary requirements for grid computing [3], so as to be adopted by application developers as one kind of the Grid infrastructure. N.A.B. Gray [4] made comparisons of Web Service, Java RMI, and CORBA solutions for demonstration applications.

Work related to the DRN system includes the SRB [7], the SRM [8]. The DRN system differs from those mentioned above in that it provides a file I/O interface to the actual data item, which may reside in a file system or a database system. Thus, the DRN system supports more types of storage resources. Till now, it has been implemented for a variety of storage systems and OS platforms, and the resource providers can implement the data accessing logic in the Resource Agent themselves. Using DRN system, users do not need to care about the communications, which is inter-realm by CORBA mechanism. 


\section{System Architecture}

We propose a novel architecture of Distributed Resource Navigation System. We developed Resource Agents (RAs) to provide seamless access to data stored on a variety of storage resources, including file systems and database systems. Furthermore, we define IDL files with file accessing operations, including readFile, writeFile, makeDir, deleteDir, etc. For each kind of resource, we implemented the object with this kind of IDL. Applications can use the distributed objects' operations to access data stored anywhere of the distributed storage sites. The RA is used to provide the capability to discover the information needed, identify data collections of interest, and select and retrieve data items that may be distributed across a wide network. The application builds on our system can use RAs to access heterogeneous storage resources in a distributed system. The DRN employs a metadata Navigation Service to manage descriptive as well as system metadata associated with data collections and resources. Using the NS, we can store and retrieve metadata about system entities, including RAs' object reference, storage resources, data items and resource access control rules. By providing location transparency, the NS enables attribute-based data accessing. This means that applications can locate the data without providing low-level details or without referencing the data by path names.

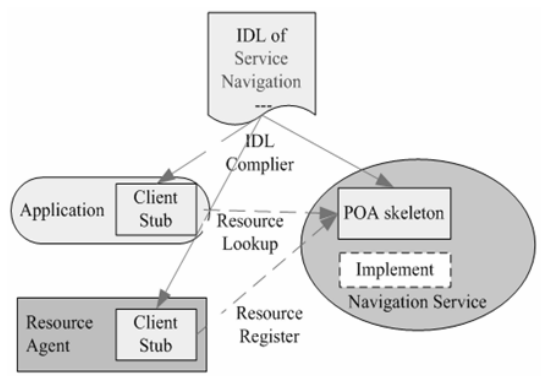

(a)

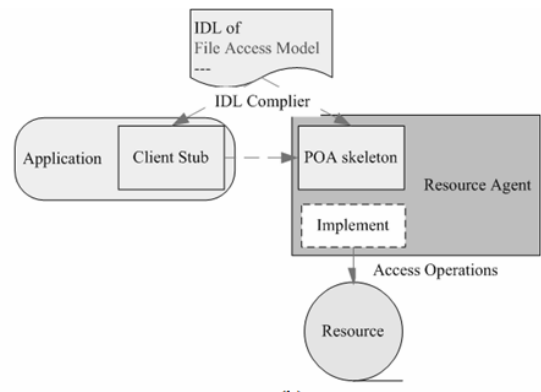

(b)

Fig. 1. Client/Server Components of (a) the Navigation Service (b) the Resource Agent

Navigation Service, as shown in Fig. 1-a, a client-side stub is auto-generated from this interface. On the server side, the interface is processed to yield a base class for the implementation class. In our DRN system, the application communicates with Services using CORBA mechanism. The application and RA use the Client Stub to call Navigation Service's functions.

By using Resource Agent, applications can seamless access the data and information stored in the DRN system. The Resource Agent works as a resource wrapper, and it provides the file model operations. From the viewpoint of the resource users, all resources are file systems. As shown in Fig. 1-b, on the client side, applications use the Client Stub, which is auto-generated from the interface, to call the remote service. On the other side, resource providers must accomplish the function implements. As CORBA is a language independent middleware, developers can use their favorite language to accomplish the job. 


\section{Experiments}

In this section we describe our implementations in using the DRN in some applications. In order to demonstrate the features of the DRN system, we present these applications with synthesis data. We also utilize the implementation to illustrate how to deploy the data management system with the DRN system. The total data size of the implementation is $400 \mathrm{~GB}$, including about 4,300 files stored in a number of file system servers, and more than 6,100 data items stored in certain number of DBMS.

One of the most important features of this architecture is that the NS must provide data source register and un-register steadily. Then we design an artificial experiment to prove this. In this experiment, that application Register-Machine use number 1 to 400 as the resource register name, it creates 20 threads register and un-register to NS circularly. Then we have application Caller to call for the resources which have registered at NS. Register-Machine will make the NS throw exception named ResAlreadyExist, and Caller will induce exception named ResNotExist. Then we compare two log file to show whether the NS can handle this.

Experimental results are shown in Fig. 2-a. The number of registered RA is the RA which has registered at NS, and the number of Called RA is the RA which has been called from Caller. The results follow our program design. Experimental results show that Navigation Service can handle parallel request steadily.

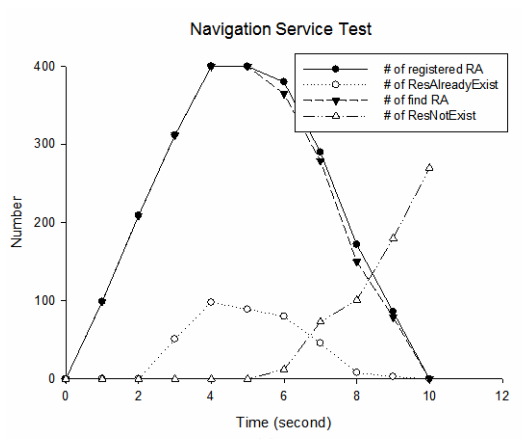

(a)

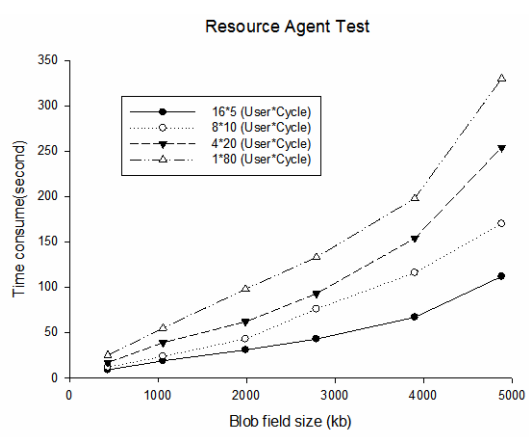

(b)

Fig. 2. Experimental Results of (a) NS, (b) RA test

Table 1. Experimental Results of many users concurrent access different blob size

\begin{tabular}{llll}
\hline $\begin{array}{l}\text { Blob Field } \\
\text { Size }(\mathrm{kb})\end{array}$ & $\begin{array}{l}\text { The number of } \\
\text { Users }\end{array}$ & $\begin{array}{l}\text { The number of } \\
\text { Cycles }\end{array}$ & $\begin{array}{l}\text { Consume } \\
\text { Time (second) }\end{array}$ \\
\hline 434 & 1 & 10 & 1 \\
434 & 5 & 10 & 7 \\
434 & 10 & 10 & 13 \\
434 & 30 & 10 & 37 \\
434 & 60 & 10 & 75 \\
434 & 100 & 10 & 123 \\
\hline
\end{tabular}


We also evaluate data access consume in our system. In this experiment we use a application simulate many users concurrently get the blob field from RA, which wrapped a database resource. We analysis the time consume with different file sizes and the time consume with different number of users.

Experimental results are shown in Fig. 2-b and Table 1, which 16 users with 5 cycles have a sharp reduce its' time consume when compare with 1 user with 80 cycles. According to this, we can conclude that RA supports concurrent access and has a good performance.

\section{Conclusion}

The DRN system provides the core service for building data management systems that span multiple administration domains, multiple types of storage systems, and multiple types of data access environments. The DRN is currently in release 1.4; we implement it by using CORBA technology, with the platform named StarBus+ [6]. Thus, the client applications and the Resource Agent are implemented for Linux, Sun Solaris, UNIX, and Windows. In order to provide quick implementation of the Resource Agent, some kinds of templates are provided as well.

This paper proposes the first steps to create resources accessing and integration architecture for data-intensive application domains. There are still many interesting issues to be further studied in DRN, such as data replication, aggregating data in containers, and I/O command aggregation via a remote proxy, etc.

\section{References}

1. Foster I, Kesselman C, Tuecke S. The anatomy of the grid: Enabling scalable virtual organizations. Int. J. Supercomputer Applications, (2001) 15(3): 200-222

2. M. Antonioletti, A. Krause, N.W. Paton. An Outline of the Global Grid Forum Data Access and Integration Service Specifications. J.-M. Pierson (Ed.): VLDB DMG 2005, LNCS 3836, pp. 71-84, (2005)

3. Snigdha Verma, Manish Parashar, Jarek Gawor, Gregor von Laszewski. Design and Implementation of a CORBA Commodity Grid Kit. GRID (2001): 2-13

4. N.A.B. Gray. Comparison of Web Services, Java-RMI, and CORBA service implementations. Australian Software Engineering Conference, (2004)

5. Reagan W. Moore. Integrating Data and Information Management. Keynote talk of International Supercomputer Conference ISC, (2004)

6. Wang H M, Wang Y F and Tang Y B. StarBus+: Distributed object middleware practice for Internet computing. Journal of Computer Science and Technology, (2005) July, 20(4): $542-551$ 